\title{
RESEARCH ON SDG FAULT DIAGNOSIS OF OCEAN SHIPPING BOILER SYSTEM BASED ON FUZZY GRANULAR COMPUTING UNDER DATA FUSION
}

\author{
Ying Zhu' ${ }^{1,2}$, Ph. D., \\ Liang Geng ${ }^{2}$, Ph. D., \\ ${ }^{1}$ School of Automation, Huazhong University of Science and Technology, Wuhan 430074, China; \\ ${ }^{2}$ School of Science, Hubei University of Technology, Wuhan 430068, China;
}

\begin{abstract}
The research work in this paper belongs to the application of granular computing, graph theory and its application in fault detection and diagnosis. It is a cross cutting and frontier research field in computer science, information science and graph theory. The results of this paper are of great significance to the application of the fault detection and diagnosis of the ocean boilers system. This research combines granular computing theory and signed directed graph, and proposes a new method of fault diagnosis, and applies it to the fault diagnosis of ocean ship boiler system.
\end{abstract}

Keywords: Granular computing; Symbolic digraph; Fault diagnosis; Fuzzy theory

\section{INTRODUCTION}

In the past few decades, the ocean boilers system has become increasingly complex, the probability of the failure of the system is increasing, and the occurrence of the fault is also more complex [1]. If the failure of the system fails to be detected in time and correctly, it may cause a major failure in the whole system and even the catastrophic consequences of the system. As a way to improve system reliability and safety, fault diagnosis technology is proposed which is based on modern sensing technology, information processing technology, computer technology, network technology, software engineering and AI. We expect to be able to infer the state according to the observation system from the current state of the system, which has extremely important practical significance to guarantee the safe operation of marine boiler system [2]. Fig. 1 is the scheme of the ship boiler system.

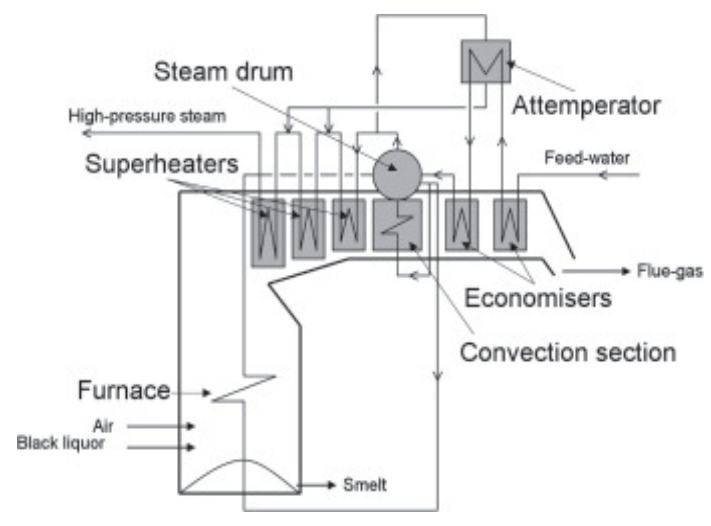

Fig. 1. Ship boiler system

When the fault diagnosis method based on the quantitative model cannot solve these problems, people start to kick the 
other path and seek other methods. The fault diagnosis method based on qualitative model is qualitative description of system structure and function by using incomplete or imprecise knowledge of the system, and qualitative reasoning is carried out to realize system fault diagnosis [3]. The qualitative model based fault diagnosis method is widely applied because of its low requirement for the analytical model of the system [4].

The symbolic directed graph is a typical method of fault diagnosis based on the qualitative model. The SDG model effectively depicts the mechanism and relationship of the system through the nodes with clear physical meaning and directed edges. It is a schema abstraction of mathematical models or physical models of processes and devices. Fault diagnosis based on SDG is based on the strong expression ability of nodes and directed edges in the SDG model, and its unique reasoning method can be used for fault detection and location quickly and effectively $[5,6,7]$.

Granular computing is a hotspot in current computational intelligence, is a new philosophical method to solve complex problems to simulate human thinking. For a complex problem, human beings have a strong global analysis ability. Granular computing can simulate human thinking from a different size (level) to abstract the perplexing problem into simpler problem. It has become a common means of solving complex problems, mass data mining, and pattern recognition. A major function of a knowledge reduction of granular computing can be under the premise of ensuring the same classification ability, remove redundant attributes decision table, minimum rules [8].

\section{INFERENCE ALGORITHM FOR SDG FAULT DIAGNOSIS}

As shown in Fig. 2 is the fault diagnosis system for the boiler system.

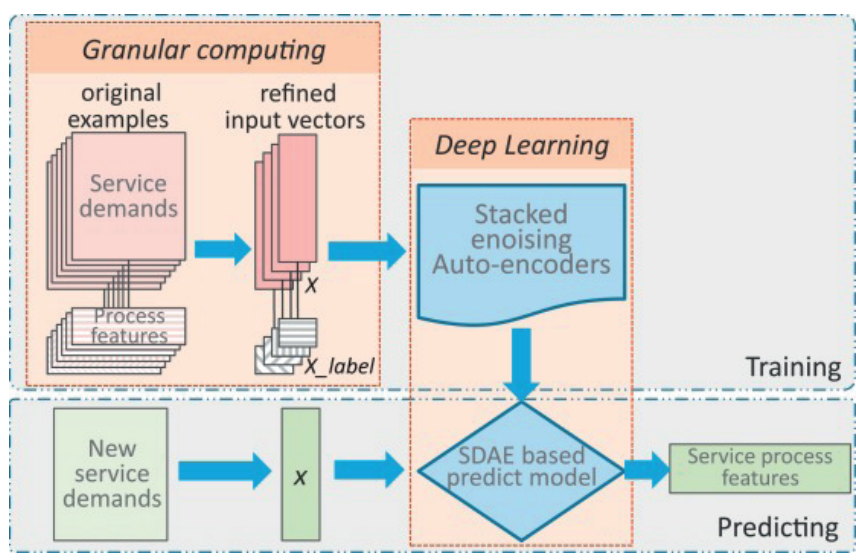

Fig. 2. Diagram for the fault diagnosis system of the boiler system

SDG is an important branch of the qualitative fault diagnosis method, at present has become a hot research topic in the field of intelligent fault diagnosis at present. Its application is more and more widely over large systems, poor real-time and low resolution defect [9].

This chapter studies the search reasoning algorithm in SDG fault diagnosis. The characteristic of this way of reasoning is that it is both logical and set theory, and therefore, it has a strong superiority in the search reasoning based on artificial intelligence [10].

Fuzzy theory is a fuzzy concept expressed by mathematical method. It uses fuzzy things and introduces quantitative information into the operation of fuzzy concepts, making the results more intuitive and objective. Fuzzy mathematics can quickly deal with a large number of matrices and vectors, which is simple and fast as shown in Fig. 2.

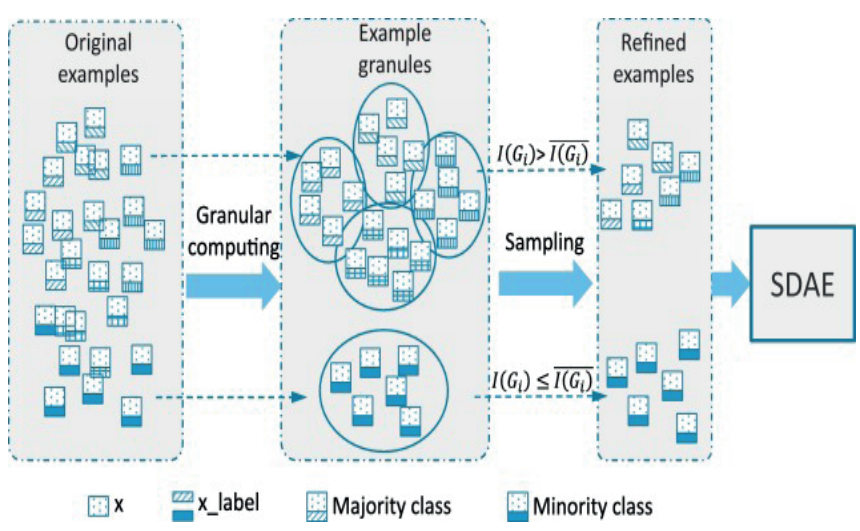

Fig. 3. Illustration of the refining examples based on granular computing

In Rough set theory, knowledge is artificially classified into objects in the field. Knowledge has granularity. So the reason for knowledge uncertainty is that knowledge granularity is too large $[11,12]$. In other words, the larger the granularity of knowledge, the more rough the information set is. In the rough set, the representation of this granular structure of knowledge is the equivalent class, and the equivalent class is obtained by the method of the equivalence relation (the method of object division) [13-15]. The elements in the equivalent class obtained from the same equivalence relation are undistinguishable, that is, the undistinguishable relation between objects.

Any one particle is a particle statement, is composed of raw sugar logic formula and its semantics [16-18].

$$
D=\left\{S\left(x_{i}, x_{j}\right) \mid x_{i}, x_{j} \in X\right\}=\left\{\lambda_{1}, \lambda_{2}, \ldots, \lambda_{m}\right\}
$$

The search reasoning of granular computing is to convert the traditional reasoning based on the rule of rule base into the grain Library in grain language.

$$
E\left[X\left(\lambda_{k}\right)\right]=\sum_{i=1}^{g} \frac{\left|G_{i}\right|}{n} * I\left(G_{i}\right)
$$


In reasoning, we can use logical method as well as set theory, which provides great convenience for reasoning process, especially in the field of artificial intelligence [19].

$$
I\left(G_{i}\right)=-\sum_{j=1}^{\left|G_{i}\right|} p_{i j} \log _{2}\left(p_{i j}\right)=\log _{2}\left(\left|G_{i}\right|\right)
$$

Transforming the rules in the traditional rule library into particle language to the decision rule particles in the grain Library [20].

$$
E\left[X\left(\lambda_{k}\right)\right]=\sum_{i=1}^{g} \frac{\left|G_{i}\right|}{n} * \log _{2}\left(\left|G_{i}\right|\right)
$$

The matching of the information particles in the field and the conditions for all the particles in the grain Library.

$$
\boldsymbol{\theta}_{e}, \boldsymbol{\theta}_{d}=\arg \min _{\boldsymbol{\theta}_{e}, \boldsymbol{\theta}_{d}} \boldsymbol{L}(\boldsymbol{x}, \boldsymbol{z})=\arg \max _{\boldsymbol{\theta}_{e}, \boldsymbol{\theta}_{d}} \boldsymbol{H}(\boldsymbol{B}(\boldsymbol{x}) \| \boldsymbol{B}(\boldsymbol{z}))
$$

The search reasoning algorithm based on similarity is in the computer stored granular library, by finding a particle that reaches a certain threshold and has the highest similarity with the collection information, it gets the corresponding decision of the information collected at the scene [21].

$$
\boldsymbol{H}(\boldsymbol{B}(\boldsymbol{x}) \| \boldsymbol{B}(\boldsymbol{z}))=-\sum_{i}\left[\boldsymbol{x}^{(i)} \log z^{(i)}+\left(1-\boldsymbol{x}^{(i)}\right) \log \left(1-\boldsymbol{z}^{(i)}\right)\right]
$$

The search reasoning algorithm for granular computing is used for fault diagnosis, and a threshold is set here:

$$
\boldsymbol{\theta}_{e}, \boldsymbol{\theta}_{d}=\arg \min _{\boldsymbol{\theta}_{e}, \boldsymbol{\theta}_{d}} \boldsymbol{J}(\boldsymbol{x}, \tilde{z})=\arg \min _{\boldsymbol{\theta}_{e}, \boldsymbol{\theta}_{d}} \boldsymbol{J}\left(\boldsymbol{x}, g_{\boldsymbol{\theta}_{d}}\left(f_{\boldsymbol{\theta}_{e}}(\tilde{\boldsymbol{x}})\right)\right)
$$

\section{DESIGN AND EVALUATION OF A SIMULATION PLATFORM FOR FAULT DIAGNOSIS}

Generally speaking, a decision table of knowledge reduction is not the only one, that there may be a number of decision table reductions of knowledge. Knowledge reduction is achieved based on decision rules in decision table and attribute reduction in number directly affects the complexity of decision rules. In the fault diagnosis, the minimum reduction can be found through the expectation of knowledge reduction.

In this section, based on the knowledge reduction algorithm of granular matrix, we propose an attribute reduction algorithm based on the importance of node attributes. After computing the kernel reduction, we add one of the most important attributes every time, until the attribute set meets the requirement of attribute reduction.

This model has 17 monitoring variables, respectively is the boiler water supply flow FR-01, superheated steam output flow FR-02, de-superheating water flow FI-03, softening water flow FR-04, gas flow, fuel oil flow rate of FR-07 and FI-06 to the catalytic deaerated water flow FI-08, furnace pressure PI-03, flue gas outlet pressure PI-05, oxygen content in flue gas AI-01, main steam high pressure gas pressure PIC-01, pressure PIC02, pressure PIC-03, liquid hydrocarbon deaerator pressure PIC-04, SAIC LIC-01, packet level of deaerator water level LIC-02 and superheated steam temperature TIC-01.

8 common failures were considered: deaerator pressure failure CI, high pressure gas pressure fault C8, liquid hydrocarbon pressure failure C7, boiler full water F2, boiler water shortage F3, boiler fire extinguishing F4, power interruption F5, temperature reducer bad F6.

3 methods are applied to attribute reduction of typical fault sample set of boiler system, and get different results. Attribute reduction algorithm which is anxious for granular matrix and node importance is reduced to 5 attributes, and the other two methods are 6 . This paper sorted the importance of the key nodes and the vulnerable nodes in the failure decision table, and used it as heuristic information to improve the efficiency of attribute reduction.

The fault 3 and the fault 9 in the system are the step changes and random changes of the temperature of the feed $\mathrm{D}$ of the reactor. After analysis, the temperature change of the feed $\mathrm{D}$ has little influence on the system variables. From the measured data, it can be seen that the temperature change of the feed $D$ has no effect on the other variables. Fault 4 and fault 11 all change the inlet temperature of the cooling water of the reactor, one is step change, one is random change, and the compatible path is consistent. Fault 14 is the viscosity of the reactor cooling water valve. When the failure occurs, the system is in a stable state. From the measured data of the system, the occurrence of the fault has no effect on other variables of the system. Therefore, only the fault $4,6,11$ of the fault occurred at various stages of the situation.

The knowledge base is the core of the expert system, which is stored and managed by the knowledge base. The rational distribution of knowledge base is beneficial to the retrieval and reasoning of knowledge, and improves the performance of the system. In this expert system, the knowledge base is composed of a text library, a picture library, and a database, which stores text, case pictures, and data tables, respectively.

The library includes: introduction of system flow, SDG reasoning process, particle similarity reasoning process, and detailed description of fault diagnosis (including the description of each node's condition, explanation of failure phenomena, and related maintenance and maintenance suggestions).

The picture library includes the core SDG diagram, the total SDG diagram, and the SDG part pattern of the fault diagnosis. 
The database includes the full node decision table, the core node decision table, the node real-time monitoring data table, the node real-time monitoring data state value table and so on.

The rule of decision table in knowledge base is composed of conditional attribute and decision attribute. Conditional attribute is the state value of corresponding node variable when the fault occurs, and the decision attribute is the fault source.

For the expert system, the beginning of the design can be as brief as possible. Need to continuously improve the rules. Before the new rule is added, it is necessary to verify whether the rule satisfies the SDG model rules, such as whether the satisfaction is added or not, and the SDG model and the node threshold setting are improved. After adding the new rule, we need to store the new all node decision table, and use the reduction algorithm of granular computing to reduce it again, and get the new core node decision table.

In addition, the domain experts also need to modify the corresponding text library and picture library corresponding to the rule.

The query of the knowledge base mainly completes the query of all kinds of files (SDG diagram, text and so on) corresponding to the fault cases.

One of the most important indicators to measure the performance of an expert system is the consistency of knowledge base. The so-called knowledge base consistency refers to the consistency of each unit in the knowledge base which affects and interconnects each other, and there is no two meaning. The rules in the knowledge base to add, delete, modify, and must carry out the rules of consistency test, especially in a large number of rules, each unit of the knowledge between the mutual influence and mutual relations will become very complex, the knowledge base consistency test is very important. The main work of the knowledge management module is to check the knowledge base to ensure the consistency and integrity of knowledge.

By calling the MATLAB program, the whole node decision table in the database is transformed into the corresponding matrix form of MATLAB, and the matrix is reduced by using the particle knowledge reduction algorithm. The matrix form of the simplified decision table is saved to the core node decision table of the database.

Fault diagnosis system is mainly implemented in King View "application command language" centralized diagnosis algorithm. The programming command language in configuration is a programming language which is grammatically similar to $C$ language. People can use these programming command languages to enhance the flexibility of applications, and do some arithmetic and operation calculations. The information table is shown in Tab.1.

Tab. 1. Information table

\begin{tabular}{|c|c|c|c|c|}
\hline & $\mathrm{a} 1$ & $\mathrm{a} 2$ & $\mathrm{a} 3$ & $\mathrm{a} 4$ \\
\hline $\mathrm{u} 1$ & 0 & 1 & 2 & 0 \\
\hline $\mathrm{u} 2$ & 1 & 2 & 0 & 2 \\
\hline $\mathrm{u} 3$ & 1 & 0 & 1 & 0 \\
\hline $\mathrm{u} 4$ & 2 & 1 & 0 & 1 \\
\hline $\mathrm{u} 5$ & 1 & 1 & 0 & 2 \\
\hline
\end{tabular}

Command languages are executed by event triggered events, such as timing, data change, keypad press, click of the mouse, and so on. According to different events and functions, the programming command language is divided into application command language, event command language, hotkey command language, data change command language, semantic function command language, animation track command language $g$ and picture command language. These are the command language for editing input through the command language editor, was compiled in „King view” operating system.

After the operation of the background operation management part, when the fault occurs, the operation result of the program will be changed significantly by using the program command language, causing the event to trigger, pop up the cause of the failure display interface, point out the source of the failure, and give the corresponding maintenance or operation suggestion.

The main fault of the system found by the system is shown in Tab. 2:

Tab. 2. Fault Information table

\begin{tabular}{|c|c|c|c|c|c|c|}
\hline Fault 1 & 1 & 0 & 0 & 1 & 0 & 0 \\
\hline Fault 2 & 1 & 0 & 0 & 1 & 0 & 0 \\
\hline Fault 3 & 1 & 1 & 0 & 1 & 0 & 0 \\
\hline Fault 4 & 1 & 1 & 0 & 1 & 1 & 0 \\
\hline Fault 5 & 1 & 1 & 0 & 0 & 0 & 1 \\
\hline Fault 6 & 1 & 1 & 0 & 0 & 0 & 0 \\
\hline Fault 7 & 1 & 1 & 1 & 0 & 0 & 0 \\
\hline Fault 8 & 1 & 0 & 1 & 0 & 0 & 0 \\
\hline Fault 9 & 1 & 0 & 1 & 0 & 1 & 0 \\
\hline Fault 10 & 0 & 0 & 1 & 0 & 1 & 0 \\
\hline
\end{tabular}

Based on the above simulation results, the three methods based on similarity reasoning, which are based on similarity reasoning, have the fastest speed of diagnosis because of their simple method and convenient reasoning. Because of the "layering" thought, the hierarchical SDG fault diagnosis only searches in the lower layer when searching for the cause of the fault. It improves the diagnostic accuracy of the system while improving the diagnostic speed. Fault diagnosis based on fuzzy reasoning, fuzzy quantitative join in qualitative SDG, the qualitative knowledge and quantitative knowledge organically six, effectively improve the resolution of fault diagnosis based on similarity reasoning is not only superior, and two kinds of SDG fault diagnosis is better than the front.

Although the above several fault diagnosis methods have advantages and disadvantages, however, a fault in the system, such as 3, 9, 13-15 fault and other five kinds of fault are the correct diagnosis results failed to give, the main reason is the five failure of the system measurement variable data fault diagnosis method proposed in this paper is very little, not suitable for on the trouble, can use the principal component analysis method of mathematical statistics for fault diagnosis. The use of principal component analysis of the five kinds of fault diagnosis results are given in detail, and the method of fault identification is 4, 5, 7 and 10 of the rate is very low, the fault diagnosis method is proposed in this paper is very 
good for the four kinds of fault diagnosis results, mainly due to a variety of variables influence the method proposed in this paper the faults were considered, including changes in reactant concentration, control variables change; fault diagnosis literature selected only 16 variables for a system of 22 continuous measurement variables for fault diagnosis. Such as fault 4, the controller under the control of the reactor temperature and reactor cooling water outlet temperature is almost constant, by continuously measuring the variables it is difficult to observe the change of system, this method takes into account the effects of the control variables, observed the change of the reactor cooling water valve V51, accurately diagnose the fault. Faults 5, 7, and 10 are similar. Therefore, fault diagnosis is a comprehensive and complex work. For a practical diagnosis system, multiple fault diagnosis methods are usually applied together to accomplish their diagnostic tasks. As shown in Fig.4, the learning efficiency of various systems are shown.

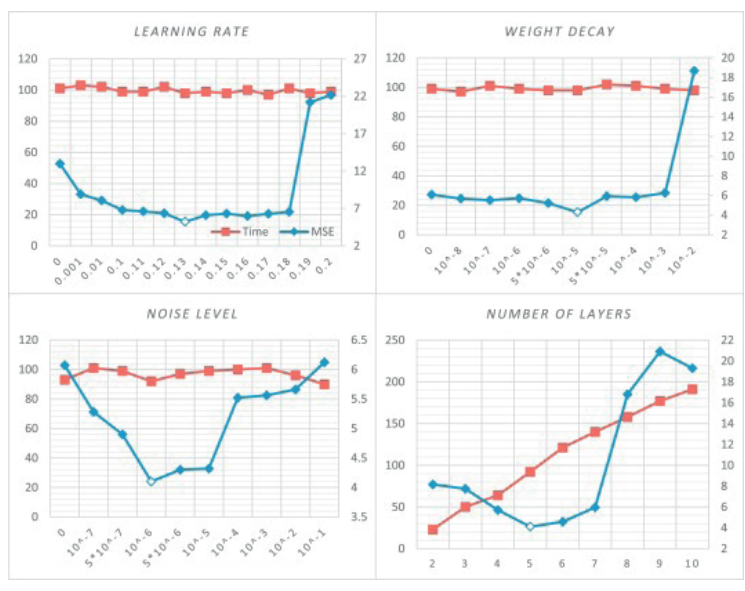

Fig. 4. Hyper-parameters setting experiments for deep neural network

\section{CONCLUSIONS}

In this paper, the principle of grain calculation and symbolic digraph is mainly studied and the application of the two groups in the fault diagnosis of the marine boilers system is also applied. Granular computing is a hotspot in current computational intelligence, is a new philosophical method to solve complex problems to simulate human thinking, the theory, rough set theory, a superset of quotient space theory. Granular computing abstracts the complicated problems from different granularity (level), and induces them into simpler problems. Then we analyze and solve these simple problems. A large function of grain computing - knowledge reduction can remove the redundant attributes of the decision table and derive the minimum rules, on the premise of ensuring that the classification ability is constant.

Sign directed graph is a kind of fault diagnosis method based on qualitative analysis, the relationship between the variables can effectively express complex system, the system has a strong relationship between the completeness of the representation is also very complicated, some redundant variables also exist in the system, affect the speed of fault diagnosis. Therefore, we introduce granular computing to fault diagnosis based on signed digraph, and propose granular computing based SDG fault diagnosis method, and do some research on some key problems.

\section{BIBLIOGRAPHY}

1. Ciabattoni, L., Ferracuti, F., Freddi, A. and Monteriu, A.: Statistical spectral analysis for fault diagnosis of rotating machines, Ieee Transactions on Industrial Electronics, Vol. 65, no. 5, pp. 4301-4310, 2018.

2. He, W., He, Y., Luo, Q. and Zhang, C.: Fault diagnosis for analog circuits utilizing time-frequency features and improved vvrkfa, Measurement Science and Technology, Vol. 29, no. 4, pp. 1-4, 2018.

3. Jack, Q., John, E. and Pan, Y.: Multi-scale stochastic resonance spectrogram for fault diagnosis of rolling element bearings, Journal of Sound and Vibration, Vol. 420, no. 2, pp. 174-184, 2018.

4. Khan, S., Gani, A., Wahab, A.W.A. and Singh, P.K.: Feature selection of denial-of-service attacks using entropy and granular computing, Arabian Journal for Science and Engineering, Vol. 43, no. 2, pp. 499-508, 2018.

5. Li, Y., Li, G., Yang, Y., Liang, X. and Xu, M.: A fault diagnosis scheme for planetary gearboxes using adaptive multi-scale morphology filter and modified hierarchical permutation entropy, Mechanical Systems and Signal Processing, Vol. 105, no. 4, pp. 319-337, 2018.

6. Wang, Y., Zheng, Y., Fang, H.-J., Wang, Y.-W.: ARMAX model based run-to-run fault diagnosis approach for batch manufacturing process with metrology delay. International Journal of Production Research, 2014, 52(10): 2915-2930.

7. Zheng,Y., Fang,H.-J., Wang,H.-O.: Takagi-Sugeno fuzzy model-based fault detection for networked control systems with markov delays. IEEE Transactions on System, Man and Cybernetics, Part B: Cybernetics, 2006, 36(3): 924-929.

8. Liu, H., Li, J., Guo, H. and Liu, C.: Interval analysis-based hyperbox granular computing classification algorithms, Iranian Journal of Fuzzy Systems, Vol. 14, no. 5, pp. 139156, 2017.

9. Lung, J., Chen, Q., Mao, N. and Jack, P.: Combining granular computing technique with deep learning for service planning under social manufacturing contexts, Knowledge-Based Systems, Vol. 143, no., pp. 295-306, 2018.

10. Micheal, J., Zi, Y., Chen, J., Zhou, Z. and Wang, B.: Liftingnet: A novel deep learning network with layerwise 
feature learning from noisy mechanical data for fault classification, Ieee Transactions on Industrial Electronics, Vol. 65, no. 6, pp. 4973-4982, 2018.

11. Pecht, M., Zhao, M., Kang, M. and Tang, B.: Deep residual networks with dynamically weighted wavelet coefficients for fault diagnosis of planetary gearboxes, Ieee Transactions on Industrial Electronics, Vol. 65, no. 5, pp. 4290-4300, 2018.

12. Sheikhian, H., Delavar, M.R. and Stein, A.: A gis-based multi-criteria seismic vulnerability assessment using the integration of granular computing rule extraction and artificial neural networks, Transactions in Gis, Vol. 21, no. 6, pp. 1237-1259, 2017.

13. Wang, J., Cheng, F., Qiao, W. and Qu, L.: Multiscale filtering reconstruction for wind turbine gearbox fault diagnosis under varying-speed and noisy conditions, Ieee Transactions on Industrial Electronics, Vol. 65, no. 5, pp. 4268-4278, 2018.

14. Wang, L., Liu, Z., Miao, Q. and Zhang, X.: Complete ensemble local mean decomposition with adaptive noise and its application to fault diagnosis for rolling bearings, Mechanical Systems and Signal Processing, Vol. 106, no. 5, pp. 24-39, 2018.

15. Wang, M., Hu, N.-Q. and Qin, G.-J.: A method for rule extraction based on granular computing: Application in the fault diagnosis of a helicopter transmission system, Journal of Intelligent \& Robotic Systems, Vol. 71, no. 3-4, pp. 445 455, 2013.

16. Wang, Q. and Gong, Z.: An application of fuzzy hypergraphs and hypergraphs in granular computing, Information Sciences, Vol. 429, no., pp. 296-314, 2018.

17. Wu, H., Liu, Y., Yan, P., Fang, G. and Zhong, J.: A frequent itemset mining algorithm based on composite granular computing, Journal of Computational Methods in Sciences and Engineering, Vol. 18, no. 1, pp. 247-257, 2018.

18. Xiahou, K.S. and Wu, Q.H.: Fault-tolerant control of doublyfed induction generators under voltage and current sensor faults, International Journal of Electrical Power \& Energy Systems, Vol. 98, no. 6, pp. 48-61, 2018.

19. Yang, S.-C., Hsu, Y.-L., Chou, P.-H., Chen, G.-R. and Jian, D.-R.: Online open-phase fault detection for permanent magnet machines with low fault harmonic magnitudes, Ieee Transactions on Industrial Electronics, Vol. 65, no. 5, pp. 4039-4050, 2018.

20. Yu, Y., Zhao, Y., Wang, B., Huang, X. and Xu, D.: Current sensor fault diagnosis and tolerant control for vsi-based induction motor drives, Ieee Transactions on Power Electronics, Vol. 33, no. 5, pp. 4238-4248, 2018.
21. Zheng, B., Li, Y.-F. and Huang, H.-Z.: Intelligent fault recognition strategy based on adaptive optimized multiple centers, Mechanical Systems and Signal Processing, Vol. 106, no. 7, pp. 526-536, 2018.

\section{CONTACT WITH THE AUTHORS}

Ying Zhu, Ph.D.

e-mail:no2kenk98@sina.com

tel.: 18971840231

School of Automation

Huazhong University of Science and Technology

Wuhan Hubei 430074

China 